\title{
Is the solar convection zone in strict thermal wind balance?
}

\author{
A. S. Brun ${ }^{1,2}$, H. M. Antia ${ }^{3}$, and S. M. Chitre ${ }^{4}$ \\ ${ }^{1}$ Laboratoire AIM, CEA/DSM-CNRS-Université Paris Diderot, IRFU/SAp, 91191 Gif-sur-Yvette, France \\ e-mail: sacha.brun@cea.fr \\ 2 LUTH, Observatoire de Paris, CNRS-Université Paris Diderot, Place Jules Janssen, 92195 Meudon, France \\ 3 Tata Institute of Fundamental Research, Homi Bhabha Road, Mumbai 400005, India \\ 4 Centre for Basic Sciences, University of Mumbai, Mumbai 400098, India
}

Received 22 August 2009 / Accepted 14 October 2009

\begin{abstract}
Context. The solar rotation profile is conical rather than cylindrical as it could be expected from classical rotating fluid dynamics (e.g. Taylor-Proudman theorem). Thermal coupling to the tachocline, baroclinic effects and latitudinal transport of heat have been suggested to explain this peculiar state of rotation.

Aims. To test the validity of thermal wind balance in the solar convection zone using helioseismic inversions for both the angular velocity and fluctuations in entropy and temperature.

Methods. Entropy and temperature fluctuations obtained from 3D hydrodynamical numerical simulations of the solar convection zone are compared with solar profiles obtained from helioseismic inversions.

Results. The temperature and entropy fluctuations in 3D numerical simulations have smaller amplitude in the bulk of the solar convection zone than those derived from seismic inversions. Seismic inversion provides variations of temperature from about $1 \mathrm{~K}$ at the surface to up to $100 \mathrm{~K}$ at the base of the convection zone while in 3D simulations they are of an order of $10 \mathrm{~K}$ throughout the convection zone up to $0.96 R_{\odot}$. In 3D simulations, baroclinic effects are found to be important to tilt the isocontours of $\Omega$ away from a cylindrical profile in most of the convection zone, helped by Reynolds and viscous stresses at some locations. By contrast the baroclinic effect inverted by helioseismology is much larger than what is required to yield the observed angular velocity profile. Conclusions. The solar convection does not appear to be in strict thermal wind balance, Reynolds stresses must play a dominant role in setting not only the equatorial acceleration but also the observed conical angular velocity profile.
\end{abstract}

Key words. Sun: interior - Sun: rotation - Sun: helioseismology - hydrodynamics - convection

\section{Introduction}

Helioseismic data from the Global Oscillation Network Group (GONG) and the Michelson Doppler Imager (MDI) have been used to infer the rotation profile in the solar interior (e.g., Thompson et al. 1996; Schou et al. 1998). The inversion results show that isocontours of the differential rotation $\Omega(r, \theta)$ are conical at mid-latitude rather than cylindrical as was expected from early numerical simulations (e.g., Glatzmaier \& Gilman 1982; Gilman \& Miller 1986). More recent theoretical work (Durney 1999; Kitchatinov \& Rudiger 1995; Brun \& Toomre 2002, hereafter BT02; Rempel 2005; Miesch et al. 2006, hereafter MBT06; Brun \& Rempel 2008; Balbus et al. 2009) indicates that in order to break the Taylor-Proudman constraint of cylindrical $\Omega$, the Sun must either have a systematic latitudinal heat transfer in its convection zone or thermal forcing from the tachocline or most likely both. This is due to the so-called thermal wind balance (Pedlosky 1987), which means that the existence in the solar convection zone of latitudinal entropy (or temperature) variation due to baroclinic effect can result in a rotation state that breaks the Taylor-Proudman constraint. Such latitudinal variations of the thermal properties at the solar surface have been looked for observationally by several groups since the late 1960's (e.g., Dicke \& Goldenberg 1967; Altroch \& Canfield 1972; Koutchmy et al. 1977; Kuhn et al. 1985, 1998; Rast et al. 2008, to cite only a few). This is a difficult task since one has to compensate for limb darkening effect, photospheric magnetic activity, instrument bias and many other subtle effects to extract a relatively weak signal (see Rast et al. 2008). In most cases a temperature contrast of a few degree $\mathrm{K}$ is found from equator to pole at the surface, the pole being warmer. In some observations a minimum at midlatitude with a warm equator and hotter polar regions is also found. The warm polar regions and cool equatorial region pattern is also found in 3D simulations of the solar convection zone with temperature variation slightly larger (i.e., of order $10 \mathrm{~K}$; BT02, MBT06). At the surface a banded structure of the temperature field (warm-cool-hot) is also found in 3D simulation of global scale convection. While very useful and instructive, most observations are confined to the solar surface and lack the information on the deep thermal structure of the solar convection zone which is key to characterise the dynamics of the deep solar convection zone. One way to remedy that limitation is to rely on helioseismic inversions that allow us to probe deeper into the Sun and to use 3D global simulations of the solar convection zone to guide our physical understanding.

Indeed, helioseismic inversions can give us the rotation rate as well as the sound speed and density in the solar interior as a function of radius and latitude. Inside the convection zone the chemical composition is uniform, and if we know the equation of state it is possible to determine other thermodynamic quantities like the temperature and entropy from the sound speed and density. Although there may be some uncertainty in the equation of state, the OPAL equation of state (Rogers et al. 1996; Rogers \& Nayafonov 2002) is quite close to the equation of state of solar material (e.g., Basu \& Antia 1995; Basu \& Christensen-Dalsgaard 1997). Consequently we use the OPAL 
equation of state to calculate the perturbations in entropy and temperature and assess how well a strict thermal wind balance is established in the solar convective envelope. To achieve this goal we make use of 2D inversions of $\Omega, S, T$, using the GONG and MDI data for the full solar cycle 23 and analyse our findings using 3D simulations obtained with the ASH (anelastic spherical harmonic) code (BT02; MBT06; Miesch et al. 2008) supported by theoretical considerations on the thermal wind balance and vorticity equations.

The paper is organised as follows: in Sect. 2 we describe the data and technique used in this work while the results for the temperature and entropy inversions are described in Sect. 3 along with those of 3D simulations. In Sect. 4 we discuss at length the thermal wind balance and its generalisation and interpret our seismic inversion with 3D simulation of global scale convection. Finally, in Sect. 5 we put our results in perspective and conclude.

\section{The helioseismic data and inversion technique}

We use data from GONG (Hill et al. 1996) and SOI/MDI (Schou 1999). Each data set consists of mean frequencies of different $(n, l)$ multiplets and the corresponding splitting coefficients. We use 130 temporally overlapping data sets from GONG, each covering a period of 108 days, starting from 1995 May 7 and ending on 2008 May 9, with a spacing of 36 days between consecutive data sets. The MDI data consist of 61 non-overlapping data sets, each covering a period of 72 days, starting from 1996 May 1 and ending on 2008 September 30. These data cover the solar cycle 23. For most of the work we use the temporal average over the available data to reduce the errors in inversion results. For this purpose we repeat the inversion process for all data sets and then take an average of all sets to get temporally averaged inversion results.

We use a 2D Regularised Least Squares (RLS) inversion technique in the manner adopted by Antia et al. (1998) to infer the angular velocity in the solar interior from each of the available data sets. Similarly, we use a 2D RLS inversion technique as described by Antia et al. (2001) to infer the sound speed and density in the solar interior. In practice, we calculate the differences $\delta c^{2} / c^{2}$ and $\delta \rho / \rho$ with respect to a reference solar model. We use the solar model from Brun et al. (2002) with tachocline mixing as the reference model. In this work, we are only interested in the latitudinal variation in solar structure inside the convection zone. Thus the fluctuation in sound speed can be converted to either temperature or entropy using the relation:

$$
\begin{aligned}
\frac{\delta c^{2}}{c^{2}}= & \frac{\delta P}{P}+\frac{\delta \Gamma_{1}}{\Gamma_{1}}-\frac{\delta \rho}{\rho} \\
= & \left(\left(\frac{\partial \ln P}{\partial \ln \rho}\right)_{T}+\frac{1}{\Gamma_{1}}\left(\frac{\partial \Gamma_{1}}{\partial \ln \rho}\right)_{T}-1\right) \frac{\delta \rho}{\rho} \\
& +\left(\left(\frac{\partial \ln P}{\partial \ln T}\right)_{\rho}+\frac{1}{\Gamma_{1}}\left(\frac{\partial \Gamma_{1}}{\partial \ln T}\right)_{\rho}\right) \frac{\delta T}{T} \\
= & \left(\left(\frac{\partial \ln P}{\partial \ln \rho}\right)_{S}+\frac{1}{\Gamma_{1}}\left(\frac{\partial \Gamma_{1}}{\partial \ln \rho}\right)_{S}-1\right) \frac{\delta \rho}{\rho} \\
& +\left(\left(\frac{\partial \ln P}{\partial S}\right)_{\rho}+\frac{1}{\Gamma_{1}}\left(\frac{\partial \Gamma_{1}}{\partial S}\right)_{\rho}\right) \delta S .
\end{aligned}
$$

Here $S$ is the specific entropy, $T$ is temperature, $P$ is pressure and $\Gamma_{1}$ is the adiabatic index. The required partial derivatives are calculated using the OPAL equation of state. The derivatives of $\Gamma_{1}$ are small in most of the convection zone, except for the ionisation zones of hydrogen and helium, but for completeness' sake we have included these derivatives in all our calculations. Helioseismic inversions for rotation and asphericity are only sensitive to the North-South symmetric components and hence the inverted profiles always show this symmetry. Hence, we show the inversion results in only one hemisphere. Actual profiles may have some asymmetry about the equator.

\section{Thermal perturbations in the solar convection zone}

Convection is a macroscopic transport of heat and energy. It is directly associated to correlations between the velocity field and temperature fluctuations (Brun \& Rempel 2008). Being able to infer the temperature and entropy perturbations in the solar convection zone is thus key to understanding its turbulent dynamics.

\subsection{The inverted profiles}

The aspherical part of temperature and entropy perturbations determined from temporally averaged GONG and MDI data are shown in Figs. 1 and 2. The maximum temperature fluctuation near the bottom of the convection zone is found to be about $100 \mathrm{~K}$. These fluctuations increase with depth initially, because of a steep increase in the temperature with depth which can induce an artificially large value for $\delta T$. The errors in $\delta T$ also increase with depth and the results may not be significant near the base of the convection zone. If we consider the relative fluctuation $\delta T / T$, then the maximum would be much closer to the surface and the value is of the order of $10^{-4}$ or less. Similarly, if the entropy fluctuation is divided by its typical value of the order of $C_{p}$, then it too would be of the same order. Both these relative perturbations are of the same order as $\delta c^{2} / c^{2}$. A detailed look at Figs. 1 and 2 reveal that the fluctuations are negative (relatively cold with respect to the spherically symmetric mean) at low latitude and warm at mid latitudes. In the bulk of the solar convection zone there is very little radial variation except near the surface. In the GONG data a cool polar region is also apparent but its significance is questionable given the relatively poor resolution of inversion techniques at high latitude. This feature is not clearly seen in the MDI data. While this latitudinal variation imprints through the surface for the entropy with little change in amplitude that is not the case for the temperature. At the surface the seismic inversion of the axisymmetric temperature fluctuations are very small which is in agreement with previous photospheric studies (Rast et al. 2008). It needs to be said that the inversions may not be reliable above the lower turning point of the modes, near the surface. Around $r=0.95 R_{\odot}$, where the inversions should be reliable, the temperature variations are of the order of $10 \mathrm{~K}$.

\subsection{The profiles realised in $3 D$ models of large scale convection}

Recent efforts to develop high resolution global simulations of the solar convection zone in order to identify the physical processes at the origin of heat, energy and angular momentum transport have been quite successful at reproducing the seismically inverted differential rotation profile (BT02; MBT06). We display in Fig. 3 a typical solution of the solar convection zone and differential rotation obtained with the ASH code (case AB3 of MBT06). We used a meridional cut to show the longitudinal and 
A. S. Brun et al: Is the solar convection zone in strict thermal wind balance?
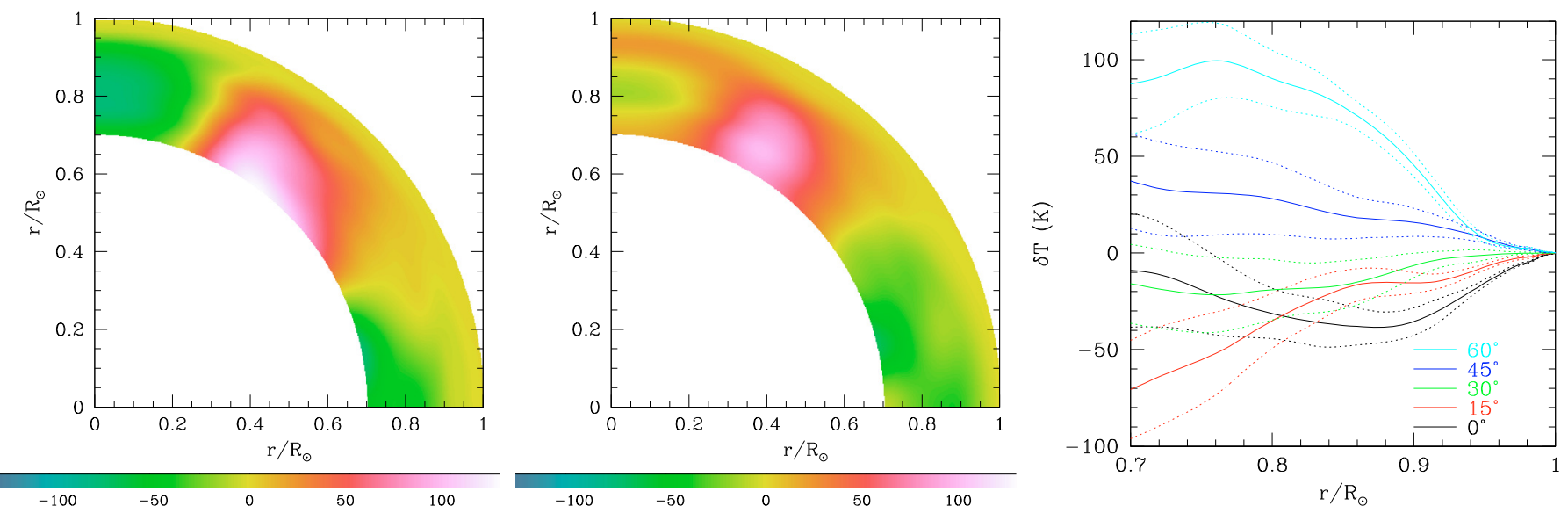

Fig. 1. The aspherical component of temperature fluctuation, $\delta T$ obtained from the temporally averaged GONG (left panel) and MDI (middle panel) data. The right panel shows the cuts at constant latitude of $\delta T$ obtained from MDI data along with $1 \sigma$ error estimates shown by dotted lines. All curves appear to merge at $r=R_{\odot}$, because $\delta T$ is of order of $1 \mathrm{~K}$ in that region.
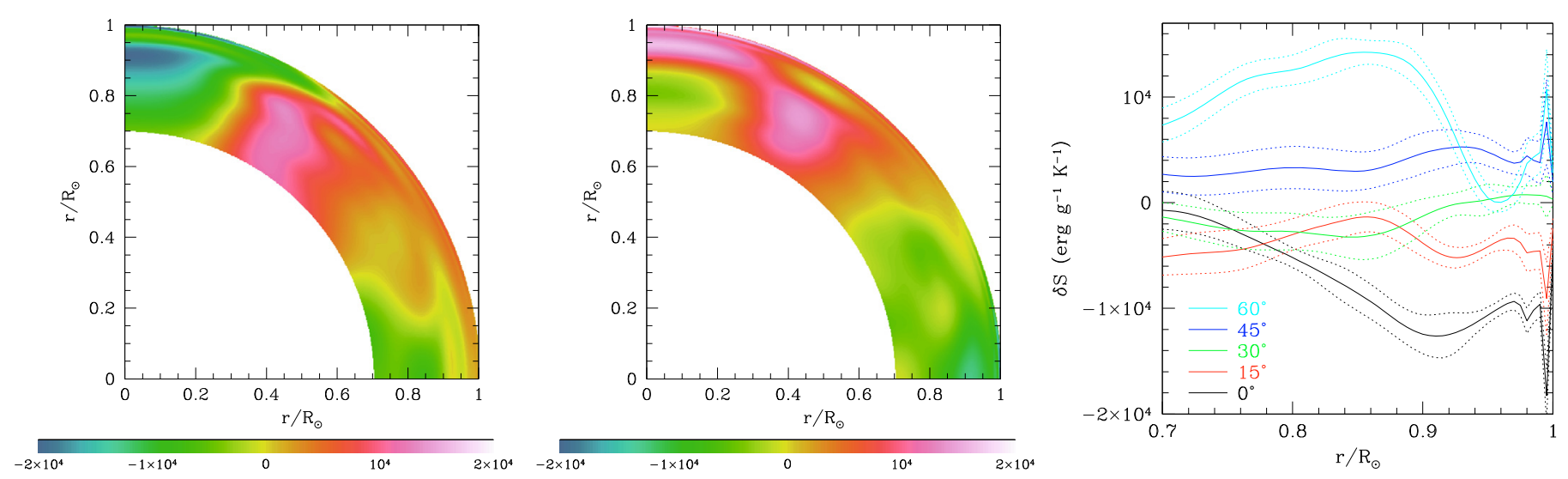

Fig. 2. The aspherical component of entropy fluctuation, $\delta S$ obtained from the temporally averaged GONG (left panel) and MDI (middle panel) data. The right panel shows the cuts at constant latitude of $\delta S$ obtained from MDI data along with $1 \sigma$ error estimates shown by dotted lines.

temporal average of the angular velocity $\Omega$ along with the temperature and entropy fluctuations with respect to a spherically symmetric background. We first note that the differential rotation in the model is solar-like, with a fast equator and slow pole, and that the iso-contours of $\Omega$ are constant along radial lines at mid-latitude (i.e., the rotation profile is conical rather than cylindrical). Its amplitude is also of the right order of magnitude. By contrast it is important to note that the temperature $T^{\prime}$ and entropy $S^{\prime}$ fluctuations ${ }^{1}$ are smaller by a factor of about 10 with respect to the seismic inversion, with temperature variations of about $10 \mathrm{~K}$ from equator to pole up to $r=0.96 R_{\odot}$. A detailed analysis of the redistribution of heat and angular momentum in the 3D models reveals that the Reynolds stresses and the latitudinal enthalpy flux are key players in establishing the profile of angular velocity and the variation of temperature as well as entropy with latitude (Brun \& Rempel 2008). Reynolds stresses transport angular momentum from the polar region down to the equator being opposed by meridional circulation and viscous effect. The heat is transported poleward by the turbulent enthalpy flux (e.g. $\bar{\rho} C_{p}\left\langle v_{\theta}^{\prime} T^{\prime}\right\rangle$, with \langle\rangle denoting an azimuthal average), $\bar{\rho}$ the mean background density and $v_{\theta}^{\prime}$ the fluctuating latitudinal component

\footnotetext{
${ }^{1}$ For the sake of clarity we make the distinction between the seismic inversion of the temperature and entropy perturbations denoted with a $\delta$ symbol and the one computed in the models denoted by a prime.
}

of the velocity field with respect to the axisymmetric mean, (see for more details Brun \& Palacios 2009) yielding a cool equator and hot poles in most of the domain. It is opposed by the thermal diffusion which tries to make the entropy and temperature field homogeneous. A careful study of the profile of the temperature and entropy fluctuations reveals that the entropy is monotonic with respect to latitude while near the surface the temperature is banded (warm-cool-hot). Furthermore the entropy profile is conical, as is the angular velocity at mid-latitude, whereas the temperature profile is more cylindrical. In these stratified (anelastic) simulations the difference between the two thermal quantities is due to density (or pressure) fluctuations that cannot be neglected. This confirms that entropy is the key quantity to consider when studying the angular velocity profile of the Sun as is clearly stated in the thermal wind equations detailed in Sect. 4.1. Mean field 2D models also find axisymmetric temperature variations of the order of a few Kelvin at the surface and in the bulk of the convection zone (Kitchatinov \& Rüdiger 1995; Küker \& Rüdiger 2005). Current global 3D numerical simulations of the solar convection zone do not model the very surface, but stop at around 0.96 to $0.98 R_{\odot}$, and as a consequence can not be used yet to model the near surface shear layer (see however the studies of Derosa et al. 2002; using a modified ASH code or of Robinson \& Chan 2001, using a spherical wedge model). 

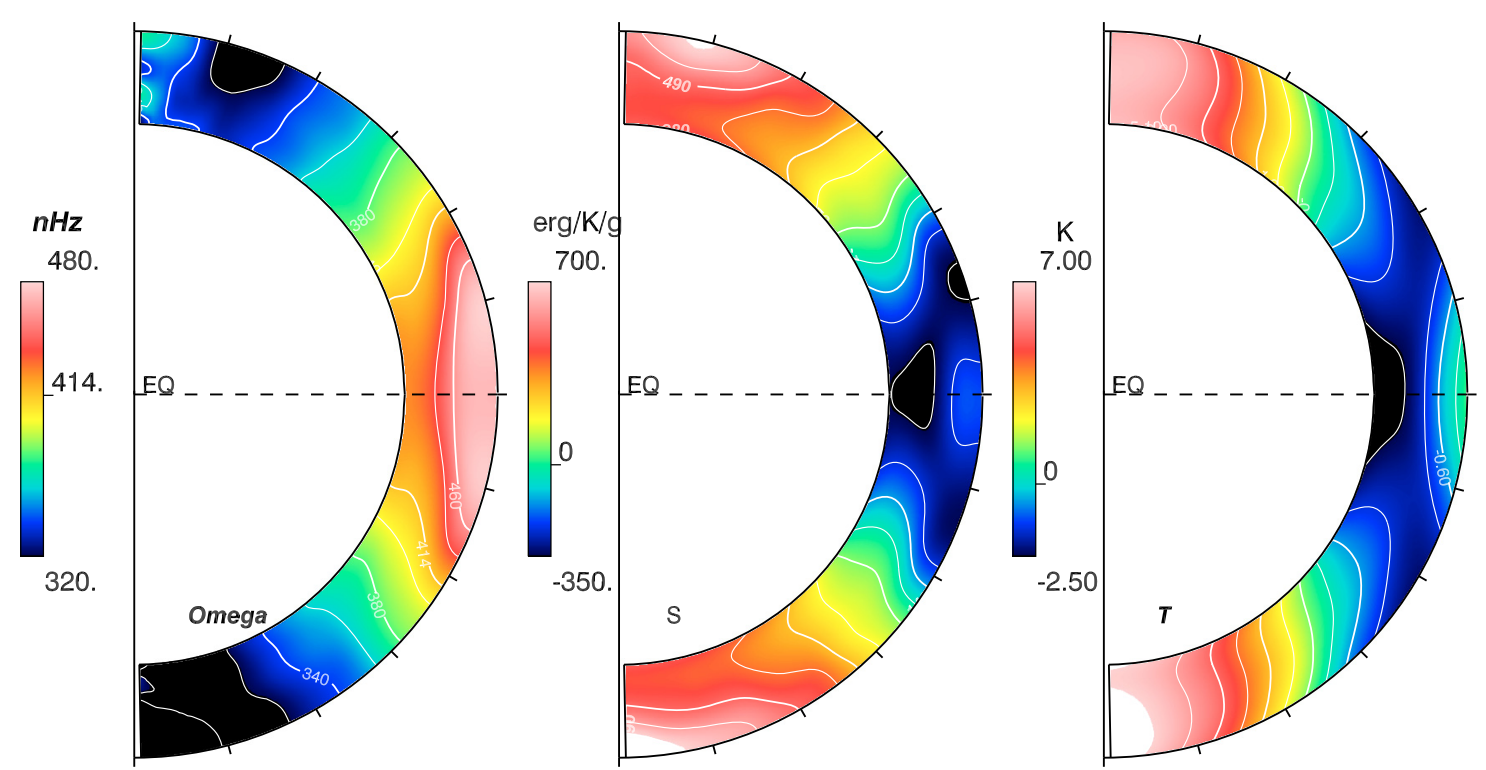

Fig. 3. Left panel: angular velocity (in $\mathrm{nHz}$ ) achieved in model $\mathrm{AB} 3$ of Miesch et al. (2006). Middle panel: associated entropy $S^{\prime}$ (erg/g/K) and temperature $T^{\prime}(\mathrm{K})$ fluctuations with respect to the spherically averaged background. All quantities have been averaged over longitude and time (10 solar periods). Note the conical profile of the angular velocity at mid latitude and the latitudinal variation of the thermal variables possessing hot poles. Near the surface the temperature is banded with warm equator, cool mid latitudes and hot poles. Contrary to the helioseismic inversion no symmetry with respect to the equator is assumed and the quantities exhibit a small North-South asymmetry.

\section{Quality of thermal wind balance achieved in the Sun and 3D models}

\subsection{Theoretical considerations}

In rotating convection, both radial and latitudinal heat transport occurs, the latter establishing latitudinal gradients in temperature and entropy within the convective zone as illustrated in Fig. 3. A direct consequence of the existence of such gradients is that the surfaces of pressure and density fluctuations will not coincide anymore, thereby yielding baroclinic effects. We can turn to the vorticity equations (Pedlosky 1987; Zahn 1992) to analyse the role of the turbulence and baroclinic effects in setting the large scale flows shown in Fig. 3. The thermal wind balance equation can be derived from the vorticity equation as discussed in detail by BT02 and MBT06. The equation for the vorticity in the purely hydrodynamical case can be derived under the anelastic approximation by taking the curl of the momentum equation (see also Fearn 1998; and Brun 2005, for its MHD generalisation and the notion of magnetic wind):

$$
\begin{aligned}
\frac{\partial \boldsymbol{\omega}}{\partial t}= & \left(\omega_{\mathrm{a}} \cdot \boldsymbol{\nabla}\right) \boldsymbol{v}-(\boldsymbol{v} \cdot \boldsymbol{\nabla}) \omega_{\mathrm{a}}-\omega_{\mathrm{a}}(\boldsymbol{\nabla} \cdot \boldsymbol{v}) \\
& +\frac{1}{\bar{\rho}^{2}} \boldsymbol{\nabla} \bar{\rho} \times \boldsymbol{\nabla} P^{\prime}-\boldsymbol{\nabla} \times\left(\frac{\rho g}{\bar{\rho}} \hat{\boldsymbol{e}}_{r}\right)-\boldsymbol{\nabla} \times\left(\frac{1}{\bar{\rho}} \boldsymbol{\nabla} \cdot \mathcal{D}\right),
\end{aligned}
$$

with $\omega_{\mathrm{a}}=\boldsymbol{\nabla} \times \boldsymbol{v}+2 \boldsymbol{\Omega}_{\mathbf{0}}$ the absolute vorticity, $\boldsymbol{\omega}=\boldsymbol{\nabla} \times \boldsymbol{v}$ the vorticity in the rotating frame, and $\mathcal{D}$ the viscous tensor given by:

$\mathcal{D}_{i j}=-2 \bar{\rho} v\left[e_{i j}-\frac{1}{3}(\boldsymbol{\nabla} \cdot \boldsymbol{v}) \delta_{i j}\right]$,

where $e_{i j}$ is the strain rate tensor, and $v$ is an effective kinematic viscosity.

This vorticity equation helps in understanding the relative importance of the different processes acting in the meridional planes. In the stationary case $\left(\frac{\partial \omega}{\partial t}=0\right)$, and assuming an azimuthal average (such that $\frac{\partial}{\partial \varphi}$ vanishes), the azimuthal component of Eq. (4) reads:

$$
\begin{aligned}
& 2 \Omega_{0} \frac{\partial\left\langle v_{\phi}\right\rangle}{\partial z}=\underbrace{-\left\langle(\boldsymbol{\omega} \cdot \boldsymbol{\nabla}) v_{\phi}-\frac{\omega_{\phi} v_{r}}{r}-\frac{\omega_{\phi} v_{\theta} \cot \theta}{r}\right\rangle}_{\text {Stretching }} \\
& +\underbrace{\left\langle(\boldsymbol{v} \cdot \boldsymbol{\nabla}) \omega_{\phi}+\frac{v_{\phi} \omega_{r}}{r}+\frac{v_{\phi} \omega_{\theta} \cot \theta}{r}\right\rangle}_{\text {Advection }} \\
& -\underbrace{\left\langle\omega_{\phi} v_{r}\right\rangle \frac{\mathrm{d} \ln \bar{\rho}}{\mathrm{d} r}}_{\text {Compressibility }}+\underbrace{\frac{1}{r}\left[\frac{\partial}{\partial r}\left(r\left\langle\mathcal{A}_{\theta}\right\rangle\right)-\frac{\partial}{\partial \theta}\left\langle\mathcal{A}_{r}\right\rangle\right]}_{\text {Viscous stresses }} \\
& +\underbrace{\frac{g}{r c_{p}} \frac{\partial\left\langle S^{\prime}\right\rangle}{\partial \theta}}_{\text {Baroclinicity }}+\underbrace{\frac{1}{r \bar{\rho} c_{p}} \frac{\mathrm{d} \bar{S}}{\mathrm{~d} r} \frac{\partial\left\langle P^{\prime}\right\rangle}{\partial \theta}}_{\text {Non adiabatic stratification }}
\end{aligned}
$$

where $\frac{\partial}{\partial z}=\cos \theta \frac{\partial}{\partial r}-\frac{\sin \theta}{r} \frac{\partial}{\partial \theta}$ and

$$
\begin{aligned}
\left\langle\mathcal{A}_{r}\right\rangle= & \frac{1}{\bar{\rho}}\left\langle\left[\frac{1}{r^{2}} \frac{\partial\left(r^{2} \mathcal{D}_{r r}\right)}{\partial r}+\frac{1}{r \sin \theta} \frac{\partial\left(\sin \theta \mathcal{D}_{\theta r}\right)}{\partial \theta}-\frac{\mathcal{D}_{\theta \theta}+\mathcal{D}_{\phi \phi}}{r}\right]\right\rangle \\
\left\langle\mathcal{A}_{\theta}\right\rangle= & \frac{1}{\bar{\rho}}\left\langle\left[\frac{1}{r^{2}} \frac{\partial\left(r^{2} \mathcal{D}_{r \theta}\right)}{\partial r}+\frac{1}{r \sin \theta} \frac{\partial\left(\sin \theta \mathcal{D}_{\theta \theta}\right)}{\partial \theta}\right]\right. \\
& \left.+\frac{1}{\bar{\rho}}\left[\frac{\mathcal{D}_{\theta r}-\cot \theta \mathcal{D}_{\phi \phi}}{r}\right]\right\rangle .
\end{aligned}
$$

In the above equation we have identified several terms:

- stretching describes the stretching/tilting of the vorticity due to velocity gradients;

- advection describes the advection of vorticity by the flow;

- compressibility describes the change of vorticity due to the flow compressibility; 

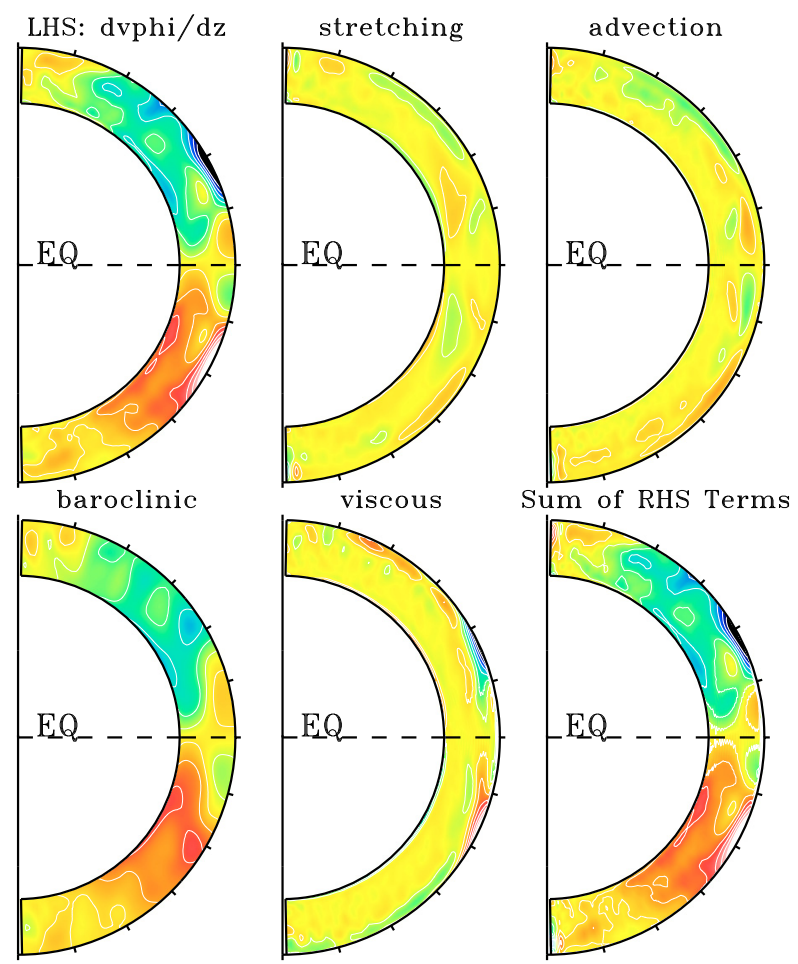

Fig. 4. Meridional cut of the terms discussed in Eq. (6) averaged over azimuth and 10 solar periods. Shown are in turn: $\partial\left\langle v_{\phi}\right\rangle / \partial z$, the stretching and advection of vorticity, the baroclinic effects, the viscous stresses and the sum of the RHS terms (we have divided all the RHS terms by $1 / 2 \Omega_{0}$ ). Each panel share the same colour table with red denoting positive value. The $\mathrm{min} / \mathrm{max}$ values used to scale the plots are $\left[-10^{-6}, 10^{-6}\right]$ expressed in $\mathrm{s}^{-1}$.

- $\frac{g}{r c_{p}} \frac{\partial\left\langle S^{\prime}\right\rangle}{\partial \theta}$ is the baroclinic term, characteristic of non-aligned density and pressure gradients;

- $\frac{1}{r \bar{\rho} c_{p}} \frac{\mathrm{d} \bar{S}}{\mathrm{~d} r} \frac{\partial\left\langle P^{\prime}\right\rangle}{\partial \theta}$ is part of the baroclinic term but arises from departure to adiabatic stratification;

- viscous accounts for the diffusion of vorticity due to viscous effects.

We wish to stress that for the nonlinear stretching and advection terms (equivalent to Reynolds stresses in Navier-Stokes equation) their azimuthal average still yields partial derivatives in $\phi$, since quadratic terms such as $\left\langle\frac{\omega_{\phi}}{r \sin \theta} \frac{\partial v_{\phi}}{\partial \phi}\right\rangle$ are non zero.

Under the assumption that the convection zone is adiabatic, the Rossby number $R_{o}=\omega / 2 \Omega_{0}$ is small, and that compressibility, Reynolds and viscous stresses can be neglected, Eq. (6) simplifies to give:

$\frac{\partial\left\langle v_{\phi}\right\rangle}{\partial z}=\frac{g}{2 \Omega_{0} r c_{p}} \frac{\partial\left\langle S^{\prime}\right\rangle}{\partial \theta}$.

This is the thermal wind equation. It simply states that baroclinic effect can break the Taylor-Proudman constraint of cylindrical differential rotation since otherwise $\partial v_{\phi} / \partial z=0$ for barotropic flows (Zahn 1992). This is due to the fact that the baroclinic terms drive meridional flows that under the influence of Coriolis force yield longitudinal flows that in turn lead to a non cylindrical state of rotation. We now turn to our numerical simulation to evaluate the role played by all the terms of the vorticity equation identified above and to discuss the quality of the thermal wind balance achieved.

\subsection{Results from $3 D$ models}

Figure 4 displays for case AB3 the left-hand side of Eq. (6), along with the dominant terms of the right-hand side and their sum. We clearly see that the sum of the dominant RHS term is in very close agreement with the LHS. We have chosen to form the temporal average over 10 solar periods because it corresponds to about 10 convective overturning times and leads to a very close balance between the LHS and the RHS of Eq. (6). Shorter averages do not lead to such a good balance, whereas longer averages change neither the quality of the balance obtained significantly nor the patterns of the various terms. Our more detailed decomposition of the vorticity equation is allowing us to identify which term is contributing and where. First the baroclinic term is found to be dominant in most of the bulk of the convection zone as was found by BT02 and MBT06. Advection terms are found to contribute both in the bulk and near the surface. Contrary to the baroclinic term they do not possess a systematic dominant contribution in each hemisphere. Their contribution leads to a change in key places, yielding a more structured profile of the RHS than the baroclinic term would have yielded if considered alone. Since the Rossby number realised in the simulation is less than one, we expect the advection and stretching term to be small on average in the simulations and indeed, their maximum amplitude is not as large as the baroclinic term. As stressed above however this is not the case in all scales nor in all locations and they do contribute in key places, leading to the very good balance shown in Fig. 4 between the LHS and RHS of Eq. (6). Finally, in our models a viscous shear layer is dominating the balance at the surface where the isocontours of $\Omega$ possess the strongest latitudinal shear. Durney (1989) and Kitchatinov \& Ruediger (1999) have also stressed that a strict thermal wind balance cannot be realised everywhere in the convection zone and that viscous stresses may play a role near the boundaries as observed in Fig. 4. We can conclude accordingly that Eq. (8) is only partly satisfied in our 3D hydrodynamical simulations of the solar convective envelope. Clearly baroclinic effects play a central role but these are far from being dominant everywhere, and considering only Eq. (8) instead of the full balance expressed in Eq. (6) would be misleading. We now turn to seismic inversion to see if the thermal wind balance is strictly realised in the Sun or if other contributions must be invoked to explain the peculiar solar rotation profile.

\subsection{Inverted solar thermal wind balance}

The entropy perturbations obtained in Sect. 3.1 can be differentiated to calculate the RHS of Eq. (8)

$\frac{g}{2 \Omega_{0} r C_{p}} \frac{\partial \delta S}{\partial \theta}$.

The result is shown in Fig. 5. We clearly see that the baroclinic term is non monotonic with respect to latitude, with large positive values near the poles and in a small region at the equator, whereas it is negative in mid latitudes. At the surface a surface thermal boundary layer is visible that yields strong radial gradients at high latitudes.

As we have done with the $3 \mathrm{D}$ model, the baroclinic term should be compared with

$\sin \theta\left(r \cos \theta \frac{\partial \Omega}{\partial r}-\sin \theta \frac{\partial \Omega}{\partial \theta}\right)$

which is shown in Fig. 6. This quantity has much less structure in the bulk of the convection zone. Except for a slightly negative 

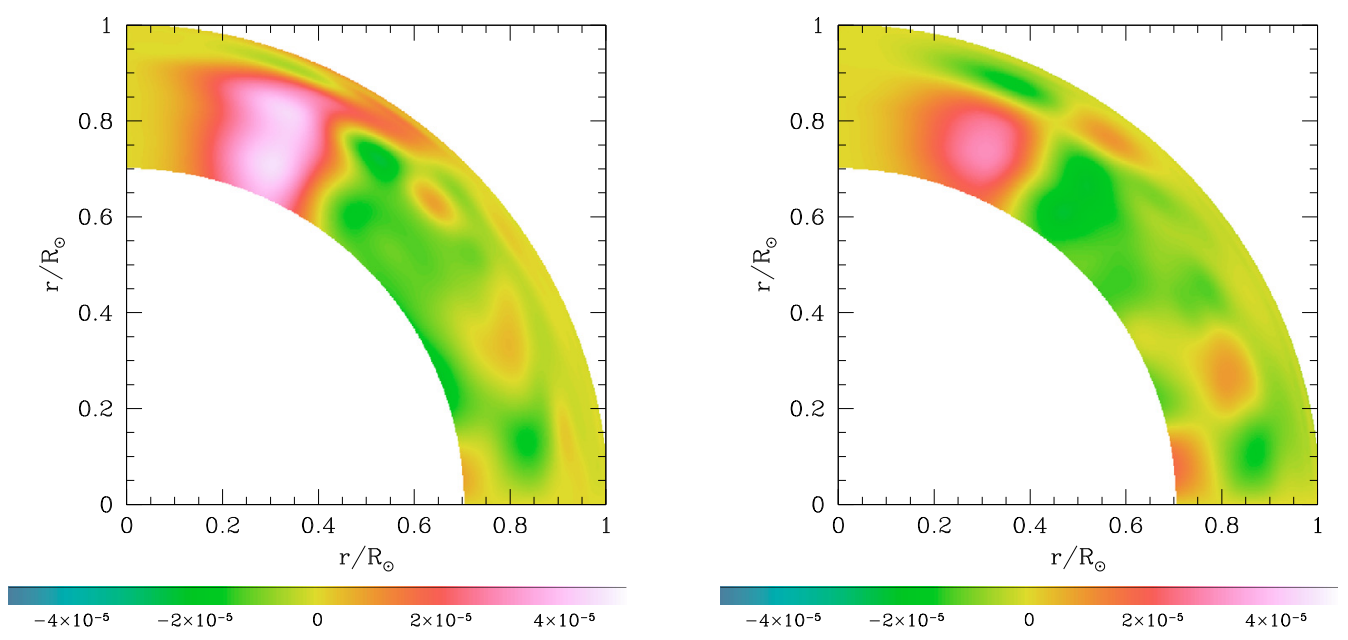

Fig. 5. The aspherical component of the latitudinal derivative of the entropy fluctuation, $\left(g / 2 \Omega_{0} r C_{p}\right)(\partial \delta S / \partial \theta)$ obtained from the temporally averaged GONG (left panel) and MDI (right panel) data. The values are in $\mathrm{s}^{-1}$.
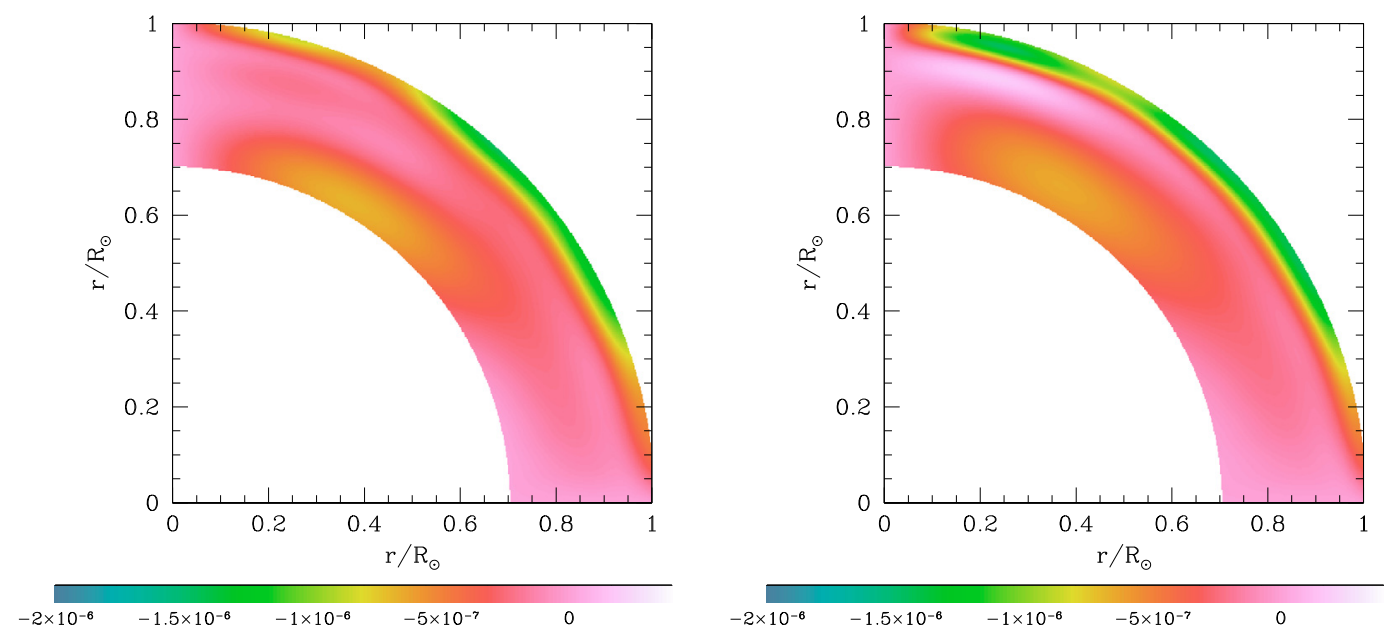

Fig. 6. The derivative of rotation velocity, $\sin \theta(r \cos \theta \partial \Omega / \partial r-\sin \theta \partial \Omega / \partial \theta)$, obtained from the temporally averaged GONG (left panel) and MDI (right panel) data. The values are in $\mathrm{s}^{-1}$.

structure at mid depth and latitude, most of the action occurs in the surface shear layer where strong negative values are found due to strong radial gradient of rotation rate in the near surface shear layer. This near surface layer is not present in the simulations and hence cannot be compared with the results of 3D simulations. It is clear that contrary to what we have seen with the 3D model in the previous section, the two quantities do not agree with each other even slightly. In fact, these two terms differ by more than an order of magnitude. While the term involving $\Omega$ in Eqs. (8) or (10) is of the same order in both the 3D simulations and the seismic inversion $\left(\sim 10^{-6} \mathrm{~s}^{-1}\right)$, this is not the case for the baroclinic terms due to the very large entropy and temperature variations in the inverted profiles. Although the $\Omega$ profile in simulations qualitatively reproduces the features seen in solar profile, the detailed latitudinal variations in the two do not match precisely.

\section{Discussion of results}

What can be the source of the disagreement between the inverted baroclinic contribution and the $z$ derivative of the angular velocity (i.e. Eqs. (8), or (9) and (10))? The first and easiest solution is that the inversion of the thermal quantities lacks the necessary accuracy, and given the increase by two orders of magnitude of the background temperature and density with depth, we end up with variations that are too large. The source of discrepancy will then be due to an overestimation of $\delta T$ and $\delta S$. It is not easy to decide if these inverted thermal fluctuations are too large or if the simulations (both 2D and 3D) underestimates the fluctuations realised in the Sun, because of for instance their limited Reynolds number. We must thus also consider the possibility that these large thermal perturbations are genuine. If this is indeed the case we need to see how we could resolve the discrepancy between the seismically inverted LHS and RHS of Eq. (8). As stated in Sect. 4.1, to obtain a strict thermal wind balance as expressed in Eq. (8), one needs to make a certain number of assumptions: adiabaticity, weak Rossby number, negligible compressibility, viscous and Reynolds stresses, stationarity. By considering further only the hydrodynamic contributions we have omitted those associated with Maxwell stresses that are certainly present in the magnetic Sun. We are confident that the Maxwell stresses are not the source of the large observed discrepancy 
because we have formed temporal averages over a maximum and minimum period of activity and the differences between the two periods are about 10 times smaller than what would be required if all the sources of discrepancy were coming from the Maxwell stresses alone. We nevertheless intend to make a more systematic study of the departure of the strict thermal wind balance linked to magnetic effects (i.e. via the so called magnetic wind) by analysing the solar cycle 23 in detail and by comparing the results with dynamo simulations of the solar convection (Brun et al. 2004). We must thus question the validity of the other hypothesis made in deriving Eq. (8). It is clearly justified to consider the viscous terms as being negligible, given the very low microscopic value of the solar kinematic viscosity. This is clearly not the case in the 3D models where near the surface they are major contributors to the overall balance (see Fig. 4, middle panel of the bottom row), but this is due to our large effective viscosity. Assuming adiabaticity is certainly reasonable in most of the convection zone, but clearly not near the surface. Since we are mostly interested in understanding the bulk dynamics of the solar convection zone, this term is indeed very small. The choice of a low Rossby number that allows us to neglect $\omega$ over the planetary vorticity $2 \Omega_{0}$, is certainly not justified at all scales of the turbulent velocity spectra, in particular for those scales much smaller than the Rossby radius of deformation (Pedlosky 1987). In the Sun the large range of convection scales implies that a subset of those scales undergo different dynamics depending on how sensitive they are to the Coriolis force. The subtle angular momentum and heat redistribution realised in the Sun is in part captured in our 3D models. We can thus analyse if the Reynolds stresses associated with the turbulent motion indeed play a central role. As discussed in detail in Brun \& Toomre (2002) and in Sect. 4 we know that it is indeed the case in our numerical simulations (see Fig. 4, middle and right panel of the top row) even though our simulations do not possess a Reynolds number and a degree of turbulence as high as that in the Sun. We can thus expect, given the very large Reynolds number of the solar convection zone, that Reynolds stresses must play a central role in the Sun in shaping the differential rotation profile and that they somehow in part compensate the baroclinic contribution to yield the observed profile of angular velocity. This is a significant result for two reasons: It confirms for one that the Reynolds stresses are key to explain the equatorial acceleration of the solar differential rotation (as anticipated by studying angular momentum transport in our simulations as in BT02 or Miesch et al. 2008). Secondly it indicates that they are also important to explain the conical profile of the solar differential rotation, helping or opposing in some regions the baroclinic effects to break Taylor-Proudman constraint. Of course this conclusion only holds if the inverted large thermal fluctuations are real.
Acknowledgements. We thank J. P. Zahn for useful comments on a draft version of this paper. We acknowledge funding by the Indian-French scientific network (IFAN). A.S.B. is grateful to the Tata Institute of Fundamental Research, Mumbai and the Indian Institute of Astrophysics, Bangalore and its director Prof. S. Hasan for their hospitality during his visit in November 2008. This work utilised data obtained by the Global Oscillation Network Group (GONG) project, managed by the National Solar Observatory, which is operated by AURA, Inc. under a cooperative agreement with the National Science Foundation. The data were acquired by instruments operated by the Big Bear Solar Observatory, High Altitude Observatory, Learmonth Solar Observatory, Udaipur Solar Observatory, Instituto de Astrofisico de Canarias, and Cerro Tololo Inter-American Observatory. This work also utilises data from the Solar Oscillations Investigation/Michelson Doppler Imager (SOI/MDI) on the Solar and Heliospheric Observatory (SOHO). SOHO is a project of international cooperation between ESA and NASA. A.S.B acknowledges funding by the European Research Council through ERC grant STARS2 207430 (www. stars2 . eu).

\section{References}

Altrock, R. C., \& Canfield, R. C. 1972, Sol. Phys., 23, 257

Antia, H. M., Basu, S., \& Chitre, S. M. 1998, MNRAS, 298, 543

Antia, H. M., Basu, S., Hill, F., et al. 2001, MNRAS, 327, 1029

Balbus, S. A., Bonart, J., Latter, H. N., \& Weiss, N. O. 2009, MNRAS, in press Basu, S., \& Antia, H. M. 1995, MNRAS, 276, 1402

Basu, S., \& Christensen-Dalsgaard, J. 1997, A\&A, 322, L5

Brun, A. S. 2005, Habilitation à Diriger les Recherches, Physics Department, University of Paris VII Denis Diderot, France

Brun, A. S., \& Palacios, A. 2009, ApJ, 702, 1078

Brun, A. S., \& Rempel, M. 2008, Space Sci. Rev., 144, 151

Brun, A. S., \& Toomre, J. 2002, ApJ, 570, 865

Brun, A. S., Antia, H. M., Chitre, S. M., \& Zahn, J.-P. 2002, A\&A, 391, 725

Brun, A. S., Miesch, M. S., \& Toomre, J. 2004, ApJ, 614, 1073

Derosa, M. L., Gilman, P., \& Toomre, J. 2002, ApJ, 581, 1356

Dicke, R. H., \& Goldenberg, H. M. 1967, Phys. Rev. Lett., 18, 313

Durney, B. R. 1989, ApJ, 338, 509

Durney, B. R. 1999, ApJ, 511, 945

Fearn, D. R. 1998, Rep. Progr. Phys., 61, 3, 175

Gilman, P., \& Miller, J. 1986, ApJS, 61, 585

Glatzmaier, G. A., \& Gilman, P. 1982, ApJ, 256, 316

Hill, F., Stark P. B., Stebbirs, R. T., et al. 1996, Science, 272, 1292

Kitchatinov, L. L., \& Rüdiger, G. 1995, A\&A, 299, 446

Kitchatinov, L. L., \& Rüdiger, G. 1999, A\&A, 344, 911

Koutchmy, S., Koutchmy, O., \& Kotov, V. 1977, A\&A, 59, 189

Kuhn, J. R., Libbrecht, K. G., \& Dicke, R. H. 1985, ApJ, 290, 758

Kuhn, J. R., Bush, R. I., Scheick, X., et al. 1998, Nature, 392, 155

Küker, M., \& Rüdiger, G. 2005, A. N., 326, 265

Miesch, M. S., Brun, A. S., \& Toomre, J. 2006, ApJ, 641, 618

Miesch, M. S., Brun, A. S., DeRosa, M. L., \& Toomre, J. 2008, ApJ, 673, 557 Pedlosky, J. 1987, Geophysical Fluid Dynamics (New York: Springer-Verlag)

Rast, M. P., Ortiz, A., \& Meisner, R. W. 2008, ApJ, 673, 1209

Rempel, M. 2005, ApJ, 622, 1320

Robinson, F. J., \& Chan, K. L. 2001, MNRAS, 321, 723

Rogers, F. J., \& Nayfonov, A. 2002, ApJ, 576, 1064

Rogers, F. J., Swenson, F. J., \& Iglesias, C. A. 1996, ApJ, 456, 902

Schou, J. 1999, ApJ, 523, L181

Schou, J., Antia, H. M., Basu, J., et al. 1998, ApJ, 505, 390

Thompson, M. J., Toomre, J., Anderson, E. R., et al. 1996, Science, 272, 1300

Zahn, J.-P. 1992, A\&A, 265, 115 